\title{
BMJ Open Impact evaluation of the free maternal healthcare policy on the risk of neonatal and infant deaths in four sub-Saharan African countries: a quasi-experimental design with propensity score Kernel matching and difference in differences analysis
}

\author{
Duah Dwomoh (D) , ${ }^{1}$ Kofi Agyabeng, ${ }^{1}$ Kwame Agbeshie, ${ }^{2}$ Gabriel Incoom, ${ }^{3}$ \\ Priscilla Nortey, ${ }^{4}$ Alfred Yawson, ${ }^{5}$ Samuel Bosomprah ${ }^{1}$
}

To cite: Dwomoh D, Agyabeng K, Agbeshie K, et al. Impact evaluation of the free maternal healthcare policy on the risk of neonatal and infant deaths in four subSaharan African countries: a quasi-experimental design with propensity score Kernel matching and difference in differences analysis. BMJ Open 2020;10:e033356. doi:10.1136/ bmjopen-2019-033356

- Prepublication history and additional material for this paper are available online. To view these files, please visit the journal online (http://dx.doi. org/10.1136/bmjopen-2019033356).

Received 01 August 2019 Revised 29 January 2020 Accepted 19 March 2020

Check for updates

(C) Author(s) (or their employer(s)) 2020. Re-use permitted under CC BY-NC. No commercial re-use. See rights and permissions. Published by BMJ.

For numbered affiliations see end of article.

Correspondence to

Dr Duah Dwomoh;

duahdwomoh@yahoo.com

\section{ABSTRACT}

Objective Despite the huge financial investment in the free maternal healthcare policy (FMHCP) by the Governments of Ghana and Burkina Faso, no study has quantified the impact of FMHCP on the relative reduction in neonatal and infant mortality rates using a more rigorous matching procedure with the difference in differences (DID) analysis. This study used several rounds of publicly available population-based complex survey data to determine the impact of FMHCP on neonatal and infant mortality rates in these two countries.

Design A quasi-experimental study to evaluate the FMHCP implemented in Burkina Faso and Ghana between 2007 and 2014.

Setting Demographic and health surveys and maternal health surveys conducted between 2000 and 2014 in Ghana, Burkina Faso, Nigeria and Zambia.

Participants Children born 5 years preceding the survey in Ghana, Burkina Faso, Nigeria and Zambia.

Primary outcome measures Neonatal and infant mortality rates.

Results The Propensity Score Kernel Matching coupled with DID analysis with modified Poisson showed that the FMHCP was associated with a $45 \%$ reduction in the risk of neonatal mortality rate in Ghana and Burkina Faso compared with Nigeria and Zambia (adjusted relative risk $(\mathrm{aRR})=0.55,95 \% \mathrm{Cl}: 0.40$ to $0.76, \mathrm{p}<0.001)$. In addition, infant mortality rate has reduced significantly in both Ghana and Burkina Faso by approximately 54\% after full implementation of FMHCP compared with Nigeria and Zambia (aRR $=0.46,95 \% \mathrm{Cl}: 0.36$ to 0.59 , $\mathrm{p}<0.001$ ).

Conclusion The FMHCP had a significant impact and still remains relevant in achieving Sustainable Development Goal 3 and could provide lessons for other sub-Saharan countries in the design and implementation of a similar policy.
Strengths and limitations of this study

- The use of more rigorous statistical methods and data from repeated cross-sectional surveys improves the robustness of the impact estimate.

- This remains the first study that has quantified the impact of free maternal healthcare policy (FMHCP) on child survival.

- Evidence from this study can be used to inform policy decisions about the implementation of FMHCP in other sub-Saharan African countries.

- Unobserved factors could bias our study results if these factors affected interventions and comparison countries in different ways.

- We cannot interpret our results as causal since the data originate from a cross-sectional study design.

\section{INTRODUCTION}

Access to primary healthcare services remains low in many low-income and middle-income countries. According to the WHO report 2017, approximately half of the world population lack access to essential health services and it is estimated that over 100 million population are still pushed into extreme poverty because of out-of-pocket health expenditure. Peters and colleagues ${ }^{1}$ as well as Jacobs and colleagues $^{2}$ have classified these factors into four main dimensions, namely geographical access, financial access, availability of healthcare and acceptability of healthcare service. Delay or lack of access to healthcare services due to financial constraints can affect child survival. Following the Abuja declaration for sub-Saharan African (SSA) countries to spend $15 \%$ of its public spending on healthcare at 
the turn of the century, Ghana in 2003 set up a National Health Insurance Scheme (NHIS) as a way of improving Universal Health Coverage. ${ }^{3}$ In September 2003, a policy exempting women in the four poorest regions of Ghana from delivery care fees was introduced by the Government of Ghana in an attempt to increase skilled birth attendance and reduce inequality in use of healthcare services. ${ }^{4}$ The policy was rolled out in all the 10 regions by the end of April 2005 but with serious challenges. Notable among them was the fact that the disbursement of funds to accredited health facilities was not forthcoming, and in October 2005 some health facilities started to charge clients again. ${ }^{4}$ In July 2008, the Government of Ghana through the NHIS implemented a national user free maternal care exemption policy to improve financial access to maternal health services and reduce maternal mortality rate, perinatal mortality rate, neonatal mortality rate (NMR) and infant mortality rate (IMR). The policy was popularly referred to as the free maternal healthcare policy (FMHCP). The main aim of the policy was to address financial barriers to demand healthcare services.

Burkina Faso is one of the countries in SSA which failed to achieve the target for Millennium Development Goal (MDG) goal number 5 (reduction of maternal mortality by $75 \%$ between 1990 and 2015). ${ }^{5}$ That notwithstanding, tremendous efforts have been made by Burkina Faso towards ensuring equitable access to maternal care services. For instance, maternal health financing and delivery reforms were developed and implemented, among which are the abolition of user fees for antenatal care (ANC) services in 2002, subsidisation of delivery costs for all women by $80 \%$ and by $100 \%$ for the poorest in 2007 and exemption of the poorest from payment of all user fees for all curative and preventive health services in $2009 .{ }^{67}$ In this article, we refer to the policy implemented in Burkina Faso as FMHCP for easy reference to countries that have implemented the intervention.

Nigeria, for instance, did not have a clear federal policy on user fees in maternal and child health, and the regional variation at the primary and secondary levels is vast. ${ }^{8}$ Although Zambia removed user fees in 2006 in rural areas only, ${ }^{910}$ the policy had not been implemented properly and no impact had been seen in the following year or two. ${ }^{11}$ That notwithstanding, fees are still payable (by cash) in urban areas and financial constraints still remain a significant barrier to institutional delivery. ${ }^{11}$ The impact of these policies, particularly on access to health services and neonatal mortality, has not been evaluated using rigorous methods, and so the empirical basis for defending these policies is weak. ${ }^{12}$ To determine the effectiveness of FMHCP in contributing to a reduction in the mortality rate relative to countries that do not have such policy, Propensity Score Kernel Matching with the difference in differences (DID) analysis was applied. Using a quasi-experimental design, the goal of this study is to determine whether the full implementation of FMHCP in Ghana and Burkina Faso contributed to the relative reduction in NMR and IMR between 2008 and
2014 compared with Nigeria and Zambia without such significant national health financing policy on maternal healthcare.

\section{METHODS AND ANALYSES \\ Data sources}

The data used in this study were obtained from 11 separate Demographic and Health Surveys (DHS) and 1 Malaria Indicator Survey (MIS). The DHS and MIS are nationally representative cross-sectional surveys which include common questions about the year of birth and survival status of all births to women of reproductive age (15-49 years). The DHS and MIS data sets are freely available and could be downloaded at the DHS website (http://dhsprogram.com) after completing the online data request registration form. With the exception of Burkina Faso that could not provide DHS but MIS data for 2014, each country contributed three different DHS data sets that were conducted between 2000 and 2014 . That is, we used the pre-baseline data from 2001/2003 to 2007/2008; baseline data 2007/2008 and end-line data $2013 / 2014$. The unit of analysis in this study is the children of women born in 5 years $(0-59$ months) preceding the survey. Detailed distribution about number of live births in 5 years preceding the survey, number of women aged 15-49 interviewed, total number of women aged 15-49 in the country at the time of the survey, year of survey and survey response rate for eligible women, NMR and IMR per 1000 live births and cumulative incidence rate per 1000 person-years at risk can be found in online supplementary appendix table S1 A.

\section{Patients and public involvement}

Patients and the public were not involved.

\section{Primary outcome measures}

The primary outcomes of interest were IMR and the NMR. In this analysis, the IMR is defined as the probability of dying between birth and first birthday whereas NMR is defined as the probability of dying between birth and the first month of life. ${ }^{13}$ All deaths that were recorded within the first 28 days after birth were coded as 1 or otherwise 0 in defining a binary indicator variable for neonatal mortality. For infant mortality, deaths within 1 year after birth in the 5 years preceding each survey were coded as 1 otherwise 0 to define a binary indicator variable for infant mortality.

\section{Exposure to FMHCP}

Countries that have abolished at least $80 \%$ of user fees for institutional delivery in SSA between the periods of 2007 and 2014 and have DHS or MIS data readily available were included in this study as intervention countries. That notwithstanding, these countries should have conducted DHS between the periods of 2000 and 2008. This was necessary to test the parallel trend assumption which is a requirement for the validity of DID design 
Table 1 Trend of neonatal and infant mortality between countries with and without FMHCP and description of the study participants: 2007/2008-2013/2014

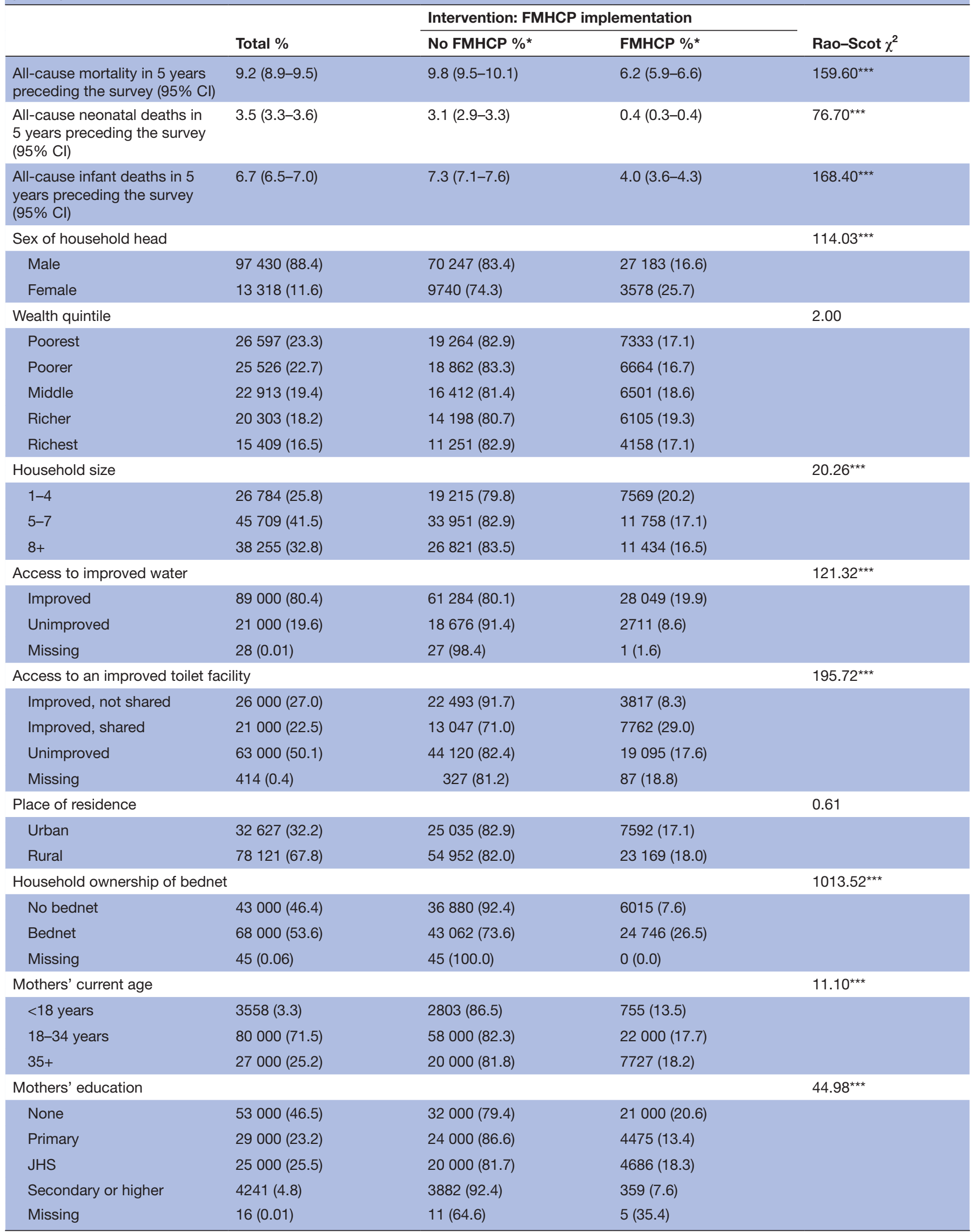


Table 1 Continued

\begin{tabular}{|c|c|c|c|c|}
\hline & \multirow[b]{2}{*}{ Total \% } & \multicolumn{2}{|c|}{ Intervention: FMHCP implementation } & \multirow[b]{2}{*}{ Rao-Scot $\chi^{2}$} \\
\hline & & No FMHCP \%* & FMHCP \%* & \\
\hline Birth order & & & & $271.39^{\star \star \star}$ \\
\hline 2nd birth & $21000(18.9)$ & $14000(76.1)$ & $7351(23.9)$ & \\
\hline 3rd birth & $17000(15.2)$ & $12000(83.1)$ & $4386(16.9)$ & \\
\hline Single & $110000(96.4)$ & $77000(82.4)$ & $30000(17.6)$ & \\
\hline Multiple & 3994 (3.6) & $2750(79.1)$ & 1244 (20.9) & \\
\hline \multicolumn{5}{|c|}{ Child mortality estimate per country } \\
\hline Country & Year of survey & NMR per 1000 live births & IMR per 1000 live births & $\begin{array}{l}\text { Cumulative incidence } \\
\text { rate per } 1000 \\
\text { person-years at risk }\end{array}$ \\
\hline \multirow{2}{*}{ Burkina Faso } & 2010 & 28 & 65 & $\begin{array}{l}44.3 \\
(40.5-48.5)\end{array}$ \\
\hline & 2014 & 27.3 & 61.4 & $\begin{array}{l}23.9 \\
(21.5-26.7)\end{array}$ \\
\hline \multirow[t]{3}{*}{ Ghana } & 2003 & 43 & 64 & $\begin{array}{l}30.0 \\
(24.2-37.7)\end{array}$ \\
\hline & 2008 & 30 & 50 & $\begin{array}{l}28.5 \\
(22.5-36.8)\end{array}$ \\
\hline & 2014 & 29 & 41 & $\begin{array}{l}15.1 \\
(11.9-19.4)\end{array}$ \\
\hline \multirow{2}{*}{ Zambia } & 2007 & 34 & 70 & $\begin{array}{l}44.7 \\
(39.1-51.4)\end{array}$ \\
\hline & 2014 & 24 & 45 & $\begin{array}{l}26.5 \\
(23.2-30.5)\end{array}$ \\
\hline
\end{tabular}

$P$ value notation: ${ }^{* * *} \mathrm{p}<0.001,{ }^{* *} \mathrm{p}<0.01,{ }^{*} \mathrm{p}<0.05$

Access to improved toilet facilities had a missing observation of $0.4 \%$.

*\% represents row percentages.

FMHCP, free maternal healthcare policy; IMR, infant mortality rate; NMR, neonatal mortality rate.

and its estimate. There were only two countries that implemented user fee reforms for maternal healthcare between 2007 and 2008. Ghana and Burkina Faso met these inclusion criteria and therefore were qualified as intervention countries. Although Zambia and Nigeria conducted DHS between 2000 and 2014, both countries did not have a universal exemption on user fees for institutional births during the study period and therefore were qualified to be used in the comparison groups. A similar study based on quasi-experimental design has provided a detailed explanation as to why Zambia, Cameroon and Nigeria could represent a valid comparison group compared with other countries in SSA in evaluating the impact of FMHCP on intermediate-term and long-term health outcomes. ${ }^{11}$ Cameroon was excluded as a comparison country in this study because there was no survey conducted in $2007 / 2008$ which represents the full policy implementation year.

Covariates assumed to be associated with child survival and included in the estimation of the propensity scores

The choice of the selected covariates in assessing risk factors of child survival was based on the analytical framework for the study of child survival in developing 
countries by Mosley and Chen. ${ }^{14}$ Specifically, we extracted data and performed the estimation of the propensity scores by using the following variables: household ownership of bednets, child's age and gender, mother's age at the time of the survey, mother's education level, household wealth, sex of the household head, urban or rural area of the household, birth order, multiple births and household size and household access to improved water and sanitation. We defined a household as having access to an improved water source if it has any of the following: piped water into the dwelling, yard or plot; public tap or standpipe, tube well or borehole; a protected dug well or protected spring; rainwater or bottled water. There is a direct correlation between access to an improved water source and infant survival. ${ }^{15}$ This analysis defines a household as having an improved sanitation if it has any of the following types of toilet facilities, and if this facility is not shared with another household: a flush or pour flush to piped sewer system, septic tank or pit latrine; a ventilated improved pit latrine; a pit latrine with a slab or a composting toilet. There is an inverse relationship between access to improved sanitation and infant mortality. Increasing access to improved sanitation is associated with lower levels of infant mortality. ${ }^{15}$ The estimation of the propensity scores was based on the binary logistic regression model that adjusted for the complex survey design structure of the data set (weighting, stratification and clustering).

\section{Statistical analyses based on DHS and MIS data sets}

Since the study pooled data from different surveys, the women's standard weights were denormalised. This was achieved by dividing the women's standard weight by the women survey sampling fraction, that is, the ratio of the total number of women aged 15-49 interviewed in the survey year over the total number of women aged $15-49$ in the country at the time of the survey. The total number of women aged 15-49 interviewed in the survey year was obtained from the DHS data sets, while the total number of women aged 15-49 years in the country at the time of the survey was obtained from our world in data (https://ourworldindata.org/). Complex survey design characteristics (weighting, stratification and clustering) were adjusted in all the analyses. In particular, we used the sampling weights in the estimation of the propensity score model and also used the sampling weight times the Kernel weight obtained from the repeated cross-section as the weight variable in the final outcome analysis. This analytic technique has been shown to produce unbiased treatment effect estimates that are generalisable to the original survey target population. ${ }^{16}$ The Kernel function used in the weight estimation was Epanechnikov and the bandwidth selection was based on cross-validation of the means of covariates. ${ }^{17}$

To determine the impact of the policy on NMR and IMR, we performed a Propensity Score Kernel Matching with DID analysis using a modified Poisson regression model with robust standard errors. We estimated the average treatment effect (ATE) using propensity scores with Kernel matching adjustment and inverse probability of treatment weighting (IPTW). The data for this study originated from multistage complex surveys and to assess the impact of the intervention, there is a need to replicate random assignment. In experimental study design with random assignment, treatment groups (countries with FMHCP) and control groups (countries with no such policy) are similar on all background characteristics (observed and unobserved) as a consequence of the randomisation, allowing for straightforward comparison of outcomes. In contrast, in complex surveys, the intervention and comparison individuals may differ significantly on background characteristics. Thus, any difference in outcomes (NMR and IMR) between the two groups may be due to these background covariates or to the intervention itself. Matching procedures, followed by regression adjustment on the matched sample, can often be a stronger approach for estimating causal effects than regression on an unmatched sample. ${ }^{18}$

The DID design is a known quasi-experimental method that is used frequently in policy evaluations to compare changes over time in a group unaffected by the policy intervention (comparison countries) with the changes over time in a group affected by the policy intervention (intervention countries) and attributes the 'DID' to the effect of the policy. ${ }^{19}$ Several sensitivity analyses were conducted to determine the robustness of our results. We tested whether the policy impact estimate is robust to the type of model specification using logit, probit and Cox proportional hazard models with robust standard errors. For the Cox model, the time-to-death with survival status as a censoring indicator was modelled. Finally, we tested whether the impact estimate is robust to different weighting procedures. First, we employed IPTW given by $w_{i}=\frac{T_{i}}{e_{i}}+\frac{1-T_{i}}{1-e_{i}}$ where $e_{k}$ is the estimated propensity score for individual $\mathrm{k}$ and $T_{i}$ is the treatment status indicator variable. The IPTW serves to weigh both the treated and control groups up to the full sample, in the same way, that surveys sampling weights weigh a sample up to a population. ${ }^{20}$ We also applied weighting by the odds to estimate the ATE on the treated (ATT) given by $w_{i}=T_{i}+\left(1-T_{i}\right) \frac{e_{i}}{1-e_{i}}$. The DID design relies on the parallel trend assumption. This assumption stated that in the absence of the intervention (FMHCP), there would be no statistically significant difference in the trend of NMR and IMR between the intervention and the comparison countries. We relied on DHS data conducted between the years 2000 and 2008 to test this assumption. P values less than 0.05 were considered as statistically significant. Data cleaning and analysis were conducted using Stata V.15 (StataCorp).

\section{RESULTS}

Results using data from 2007 to 2014 showed that approximately $9.2 \%$ (95\% CI: 8.9 to 9.5) of the 110748 children in our sample died before reaching age 5 . Within the same 


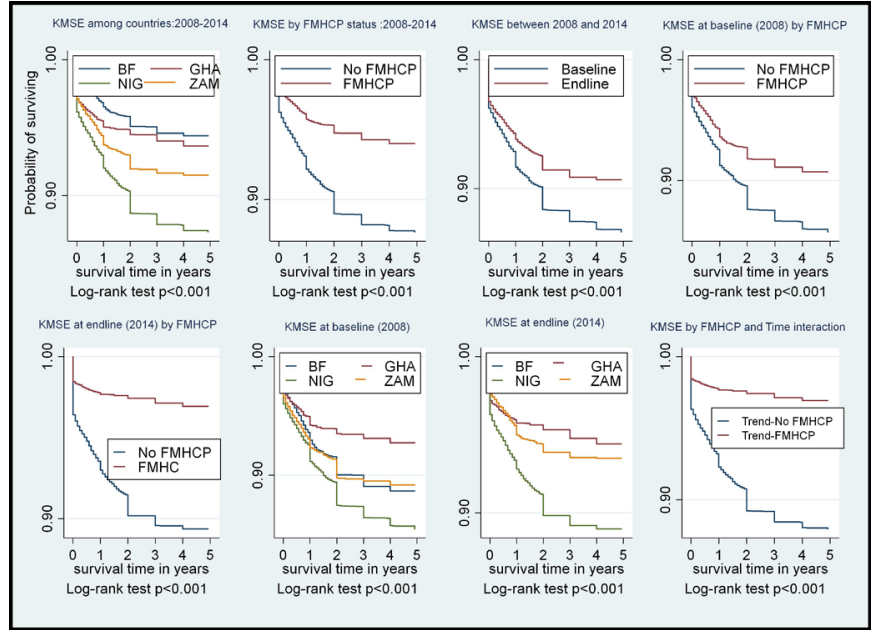

Figure $1 \mathrm{KMSE}$ at varying time points of $\mathrm{FMHCP}$ implementation. BF, Burkina Faso; FMHCP, free maternal healthcare policy; GHA, Ghana; KMSE, Kaplan-Meier survival estimate; NIG, Nigeria; ZAM, Zambia.

period, there was a statistically significant difference in the proportion of deaths between countries with FMHCP and those with no such policy (FMHCP $=6.2 \%$ (95\% CI: 5.9 to 6.6 ); no FMHCP=9.8\% (95\% CI: 9.5 to 10.1 ), RaoScot $\chi^{2}$ test $=159.6 ; \mathrm{p}<0.001$, table 1$)$. The proportion of infant deaths was $6.7 \%$ (95\% CI: 6.5 to 7.0$)$. Among countries with FMHCP, the proportion of infant deaths was approximately $4.0 \%$ (95\% CI: 3.6 to 4.3 ) compared with countries with no FMHCP where infant deaths were $7.3 \%$ (95\% CI: 7.1 to 7.6 ) and the difference was statistically significant $\left(\right.$ Rao-Scot $\chi^{2}$ test $=168.4 ; \mathrm{p}<0.001$, table 1$)$. The overall proportion of neonatal deaths was $3.5 \%$ (95\% CI: 3.3 to 3.6). FMHCP countries recorded $0.4 \%$ (95\% CI: 0.3 to 0.4 ) neonatal deaths compared with $3.1 \%$ (95\% CI: 2.9 to 3.3 ) recorded by countries with no FMHCP (Rao-Scot $\chi^{2}$ test $\left.=76.7, \mathrm{p}<0.001\right)$.

NMR and IMR per 1000 live births decline between 2008 and 2014 in both FMHCP and non-FMHCP countries but the decline was steeper at all times in the FMHCP countries at various time points (figure 1).

\section{Results on balancing and common support diagnostics of the Kernel-based matching}

Balancing tests based on standardised mean difference and ratio of variances of the observed covariates between the two sets of countries (FMHCP and non-FMHCP) were conducted before and after Kernel-based matching. This was done to ascertain how the matching procedure has reduced biases in the means and variances of the observed covariates between FMHCP countries and non-FMHCP countries. The mean difference in the observed covariate between FMHCP and non-FMHCP countries reduced significantly after matching, making the two groups as similar as possible (online supplementary appendix table S1 B). The ratio of variances in the covariate between the two sets of countries was closer to 1 after matching than before matching (online supplementary appendix table S1 C). The results showed that the propensity score

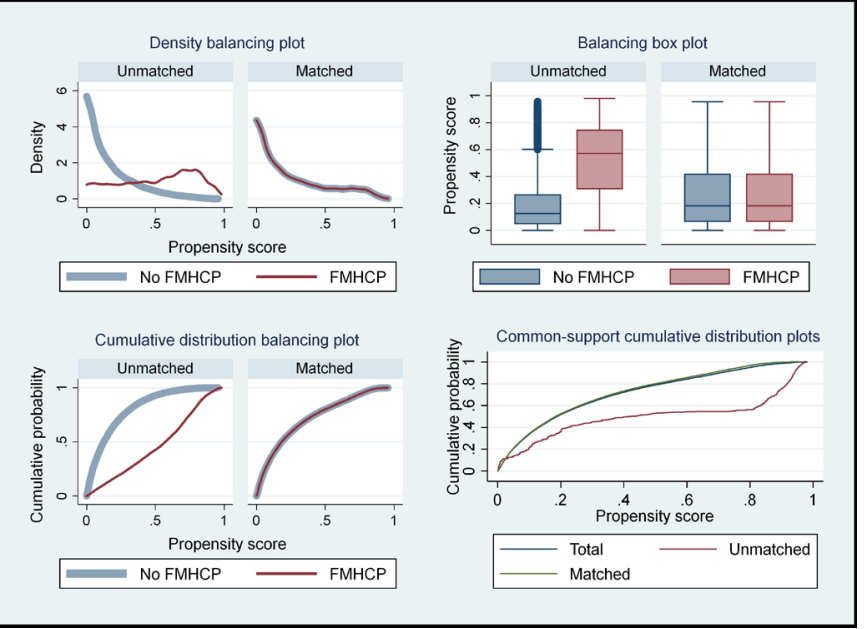

Figure 2 Balancing the diagnostic test of the Kernel-based propensity score matching. FMHCP, free maternal healthcare policy.

with Kernel-based matching reduced covariate imbalance between countries with and without FMHCP. The results from the Kernel density, cumulative distribution and the box-whisker plots in figure 2 showed that matching has made FMHCP and non-FMHCP countries more similar in terms of the observed covariates, hence any change in the risk of neonatal and infant deaths could be attributed to FMHCP.

\section{Results on the test of the parallel trend assumption}

The fixed-effects model controls for all time-invariant differences between the individuals and the countrylevel factors such as differences in geographical location, so the estimated coefficients of the fixed-effects models cannot be biased because of omitted time-invariant characteristics.

The test of parallel trends showed that after controlling for baseline individual and country time-fixed effect characteristics, maternal, child and household characteristics including household ownership of bednet, both IMR and NMR did not differ between countries with FMHCP and those with no FMHCP before the implementation of FMHCP (NMR: aRR=0.91, 95\% CI 0.71 to 1.16 ; $\mathrm{p}>0.05$; table 2).

Mortality rates were declining in all of the study countries during this time period (NMR: aRR $=0.88,95 \%$ CI: 0.75 to 1.02 ; IMR: $\mathrm{aRR}=0.84,95 \% \mathrm{CI}$ : 0.76 to 0.94 , $\mathrm{p}<0.05$, table 2), but there was no evidence of trends being different between countries that have implemented FMHCP and comparison countries. In conclusion, the parallel trend assumption was not violated and therefore estimates from DID analyses were valid.

\section{Impact of FMHCP on the risk of neonatal deaths}

The results from the modified Poisson with DID using Propensity Score Kernel Matching showed that FMHCP is associated with $45 \%$ reduction in the risk of NMR in Ghana and Burkina Faso compared with Nigeria and Zambia $(\mathrm{aRR}=0.55$, 95\% CI: 0.40 to $0.76, \mathrm{p}<0.001$, 
Table 2 Test of parallel trends assumption: risk of neonatal and infant mortality prior to free maternal healthcare policy implementation (2001-2008): modified Poisson model with robust SE on the unmatched sample

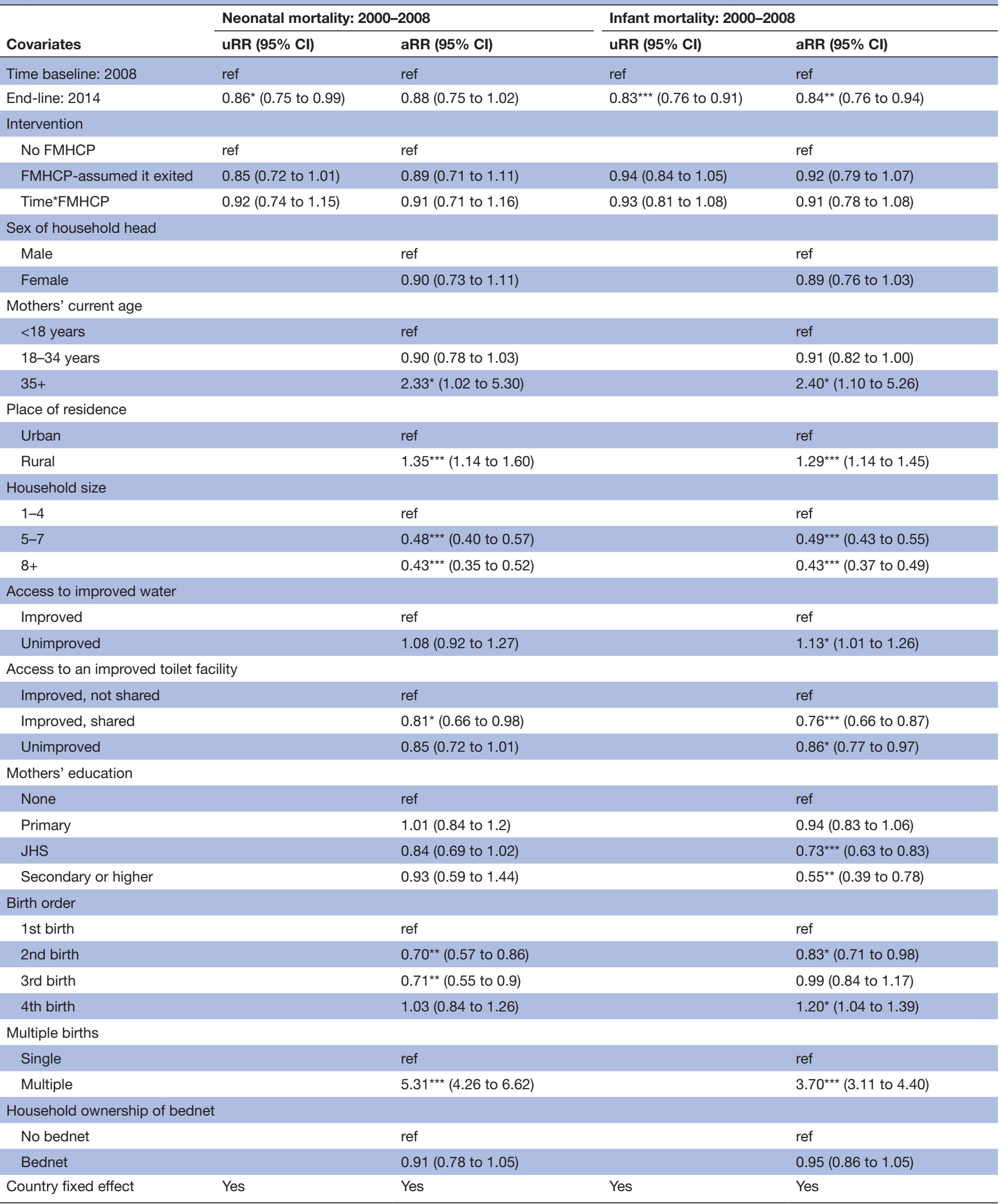

P value notations: ${ }^{* \star *} p<0.001,{ }^{* *} p<0.01,{ }^{*} p<0.05$.

With respect to Burkina Faso, 2010 demographic health survey data were used since they did not conduct any survey in 2008.

aRR, adjusted relative risk; FMHCP, free maternal healthcare policy; JHS, junior high school; uRR, unadjusted relative risk. 
table 3). Sensitivity analyses based on different outcome model specification showed similar results (table 3 ).

\section{Impact of FMHCP on the risk of infant deaths}

IMR has reduced significantly in both Ghana and Burkina Faso by approximately $54 \%$ after full implementation of FMHCP compared with Nigeria and Zambia $(\mathrm{aRR}=0.46$, $95 \%$ CI: 0.36 to $0.59, \mathrm{p}<0.001$; table 4 ). The series of sensitivity analysis that was conducted showed a similar impact on FMHCP(table 4). The analysis was adjusted for sex of the household head, mothers' current age, mothers' educational level, place of residence, wealth quintile, access to improved water and sanitation, birth order, multiple births and household ownership of bednet and country fixed effect.

\section{DISCUSSION}

This study quantified the contribution of FMHCP implementation in Ghana and Burkina Faso in the reduction of NMR and IMR. Child mortality within the implementation period in these two countries was compared with mortality in Nigeria and Zambia which do not have a significant major health financing reform in the period under consideration. It remains among the few studies to have compared the effectiveness of FMHCP in the four SSA countries using the more rigorous matching procedure with DID. Our impact evaluation found that the implementation of FMHCP led to a substantial reduction in both neonatal and infant mortality. This finding is consistent with what has been reported previously in the literature based on similar analytic technique. ${ }^{21}$ Although all the four countries studied did not attain the MDG 4, Ghana and Burkina Faso have seen a tremendous decline in the trend of NMR and IMR over the years. FMHCP was associated with substantial statistically significant reductions in IMR and NMR when these estimates were compared between Zambia and Nigeria.

It is estimated that the effective implementation of key maternal and child healthcare interventions could prevent up to $70 \%$ of neonatal deaths globally. ${ }^{22} 23$ The advantages of increasing access to facility delivery, prenatal and postnatal care through FMHCP are well documented in the literature. ${ }^{12}{ }^{24}$ FMHCP contributes greatly to increased coverage of routine immunisation as women who visit and deliver in recommended health facilities were more likely to benefit from early immunisation. The policy also promotes early and accurate diagnosis of childhood illnesses after delivery and within the postpartum period. Education on malaria preventive measures after delivery and the administration of intermittent preventive treatment for pregnancy during antenatal are a few of the benefits women derived from the policy. The FMHCP is associated with high ANC attendance and institutional delivery by skilled attendants (midwives, nurses and doctors) at the time of delivery which consequently reduced neonatal deaths and to a larger extent infant mortality. ${ }^{26}$ Increasing access to the skilled birth attendant and emergency obstetric care is accepted as the most crucial intervention for reducing maternal and newborn deaths. ${ }^{27}$

\section{Strengths and limitations}

This study has several strengths and some limitations. The advantages of using DHS as our primary source data have been well documented..$^{28}$ Paramount among these several advantages include high response rates, national coverage, high-quality interviewer training, standardised data collection procedures across countries and consistent content over time, allowing comparability across populations cross-sectionally and over time. The use of DID models with Propensity Score Kernel Matching is seen as strong non-experimental study design options when randomisation is not feasible and provides more robust inference. ${ }^{19}$

The limitation of this study originates from the fact that the DID analytical technique is generally less robust than the randomised design even though the study established that the parallel trend assumption was not violated. Although Kernel matching maximises the chance of matching control to a treated individual, observations outside the range of common support are still discarded which could potentially reduce the sample size.

We highlighted the fact that our study could still suffer from the omission of important time-varying unobserved characteristics such as total annual health expenditure could bias our study results if the omitted variables affected Ghana, Burkina Faso and comparison countries in different ways. The reason is that DID attributes to the FMHCP policy intervention differences in mortality trends between the Ghana and comparison countries that occur from the time intervention begins (2008). If any other factor is present which affect the difference in trends between the two groups differently, then the estimate from DID could be biased. In particular, health funding sources like the US President Malaria Initiative (PMI), President's Emergency Plan for AIDS Relief (PEPFAR) and the Global Fund for HIV, tuberculosis and malaria are few of the foreign aid that could have an impact on child mortality. ${ }^{29}$ For instance, Ghana and Zambia received funding support from PMI in 2008 but Burkina Faso has never benefited from PMI and Nigeria which only received funding from the PMI in 2011. Three out of the four countries studied continue to be benefited from PEPFAR but received the support at different times (Ghana; 2007, Burkina Faso; not at all, Zambia and Nigeria in 2004). The countries Ghana and Zambia still remain among the countries studied that have had the benefits of the US PMI since 2008 which also coincides with the year in which FMHCP policy became fully operational. The observed differentials among the four countries relative to foreign aid could impact on child mortality differently and bias the results.

With regards to Zambia and Nigeria, these two countries might not have a nationwide FMHCP but it is possible that there may be country-specific interventions put in place to curb the menace of child mortality. 


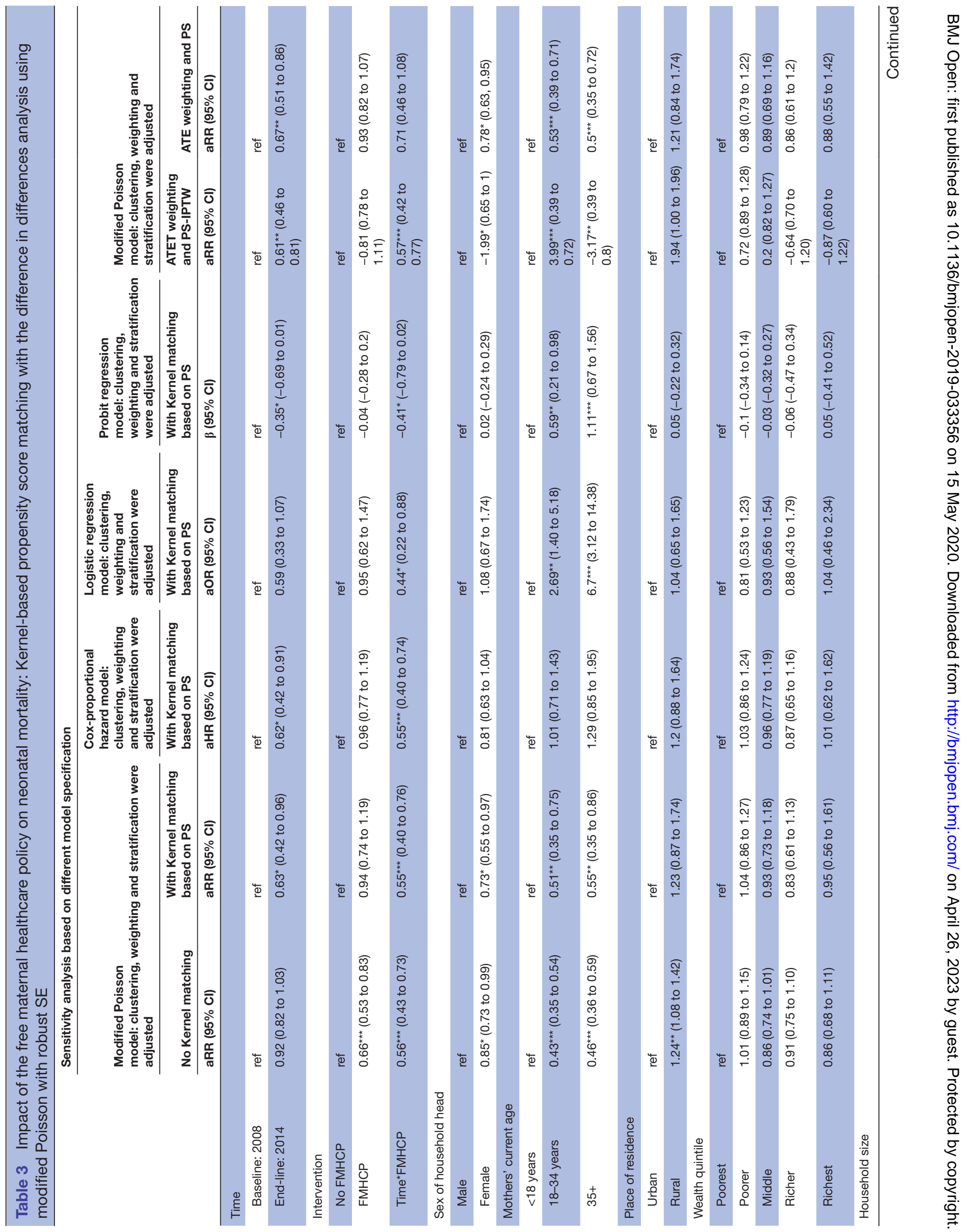


Even among the intervention countries, there may be other specific interventions that are tailored towards child mortality but were not controlled in the current study. For instance, the 'Rapid Scale-Up' programme in Burkina Faso has a component that focuses on integrated community case management and this policy has been found to reduce neonatal mortality by $6.2 \%{ }^{30}$ Other interventions such as user fee exemption and mass radio campaigns have all been found to be associated with an increase in the healthcare utilisation among children under 5 in Burkina Faso which could have a direct positive impact in reducing NMR. ${ }^{31}{ }^{32}$ In addition to the aforementioned interventions, it is worth emphasising that both Ghana and Burkina Faso receive support from the Global Fund in the fight against malaria, tuberculosis and HIV since 2003 and this might have contributed to why Burkina Faso and Ghana might be doing better in terms of reducing IMR and NMR. Despite the fact that our impact estimate of the policy may be imprecise and should be interpreted cautiously, we emphasised that the introduction of the FMHCP is associated with the reduction in both NMR and IMR which is an encouraging finding and an important contribution to the literature on the colossal benefits of FMHCP. DID still remains one of the robust quasi-experimental design to evaluate the impact of health intervention using cross-sectional timeseries data as it was the case in this study.

\section{Policy implications}

The findings from the study provide imperative evidence of an accelerated decline in child mortality rates after the introduction of FMHCP in the two West African countries. The additional investments in health tailored towards FMHCP implementation have yielded positive impacts. The implementation of the policy has reduced the financial burden associated with antenatal and postnatal care attendance and institutional delivery. Future studies should explore whether the investments made through FMHCP have spillover effects beyond the usual benefits associated with the policy, such as women empowerment, higher investment in the private sector, higher school attainment and increase in employment rate which might, in turn, lead to greater economic development. As the population of women keeps increasing geometrically in SSA, Governments should consider an alternative source of financing to sustain the policy.

\section{CONCLUSION}

The motivation of this study is to obtain more reliable evidence of how the implementation of the FMHCP in certain countries in the SSA has reduced child mortality compared with countries in the subregion with no such national policy. Our findings highlight the importance of FMHCP implementation in reducing the risk of neonatal and infant mortalities. We recommended that a similar policy should be implemented in other lower-income and 


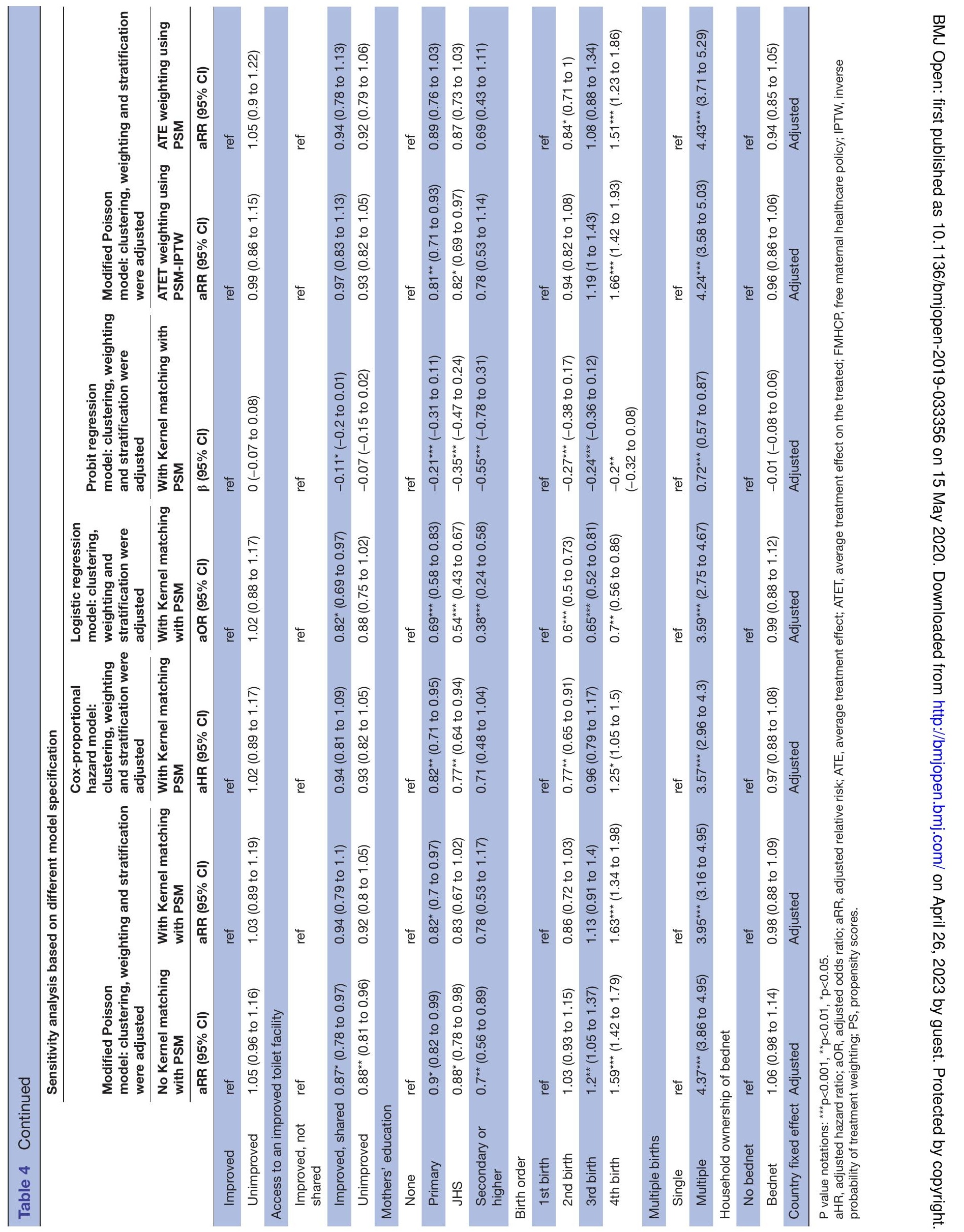


middle-income SSA countries to reduce the prevalence of neonatal and infant deaths.

\section{Author affiliations}

${ }^{1}$ Biostatistics, School of Public Health, University of Ghana College of Health

Sciences, Accra, Greater Accra, Ghana

${ }^{2}$ Municipal Health Directorate, Ghana Health Service, Eastern Region, Somanya, Ghana

${ }^{3}$ Department of Management Science, School of Business, Ghana Institute of Management and Public Administration, Accra, Ghana

${ }^{4}$ Epidemiology, University of Ghana School of Public Health, Accra, Greater Accra, Ghana

${ }^{5}$ Community Health, School of Public, College of Health Sciences, University of Ghana, Legon, Greater Accra, Ghana

Acknowledgements The authors duly acknowledge the Demographic and Health Survey Program funded by the U.S. Agency for International Development (USAID), other donors and Ghana Statistical Service for making their data available to the public. We do acknowledge all mothers who were interviewed.

Contributors DD, KwA and PN conceived and designed the study. Data management and data cleaning were done by DD, Gl and KoA. Statistical methods were drafted by DD, SB and Gl. DD, PN, Gl, KwA, KoA, SB and AY revised the draft critically. All authors have read and approved the final manuscript.

Funding The authors have not declared a specific grant for this research from any funding agency in the public, commercial or not-for-profit sectors.

Competing interests None declared.

Patient and public involvement Patients and/or the public were not involved in the design, or conduct, or reporting, or dissemination plans of this research.

Patient consent for publication Not required.

Provenance and peer review Not commissioned; externally peer reviewed.

Data availability statement Data are available in a public, open access repository. All data sets are public data and are freely available upon request.

Open access This is an open access article distributed in accordance with the Creative Commons Attribution Non Commercial (CC BY-NC 4.0) license, which permits others to distribute, remix, adapt, build upon this work non-commercially, and license their derivative works on different terms, provided the original work is properly cited, appropriate credit is given, any changes made indicated, and the use is non-commercial. See: http://creativecommons.org/licenses/by-nc/4.0/.

\section{ORCID iD}

Duah Dwomoh http://orcid.org/0000-0002-2726-9929

\section{REFERENCES}

1 Peters DH, Garg A, Bloom G, et al. Poverty and access to health care in developing countries. Ann N Y Acad Sci 2008;1136:161-71.

2 Jacobs B, Ir P, Bigdeli M, et al. Addressing access barriers to health services: an analytical framework for selecting appropriate interventions in low-income Asian countries. Health Policy Plan 2012;27:288-300.

3 Wang H, Otoo N, Dsane-Selby L. Ghana National Health Insurance Scheme: improving financial sustainability based on expenditure review. The World Bank, 2017.

4 Witter S, Arhinful DK, Kusi A, et al. The experience of Ghana in implementing a user fee exemption policy to provide free delivery care. Reprod Health Matters 2007;15:61-71.

5 Organization WH, UNICEF. Trends in maternal mortality: 1990-2015: estimates from who, UNICEF, UNFPA, world bank group and the United nations population division, 2015.

6 Belaid L, Ridde V. An implementation evaluation of a policy aiming to improve financial access to maternal health care in Djibo district, Burkina Faso. BMC Pregnancy Childbirth 2012;12:143.
7 Ridde V, Richard F, Bicaba A, et al. The National subsidy for deliveries and emergency obstetric care in Burkina Faso. Health Policy Plan 2011;26 Suppl 2:ii30-40.

8 Okonofua F, Lambo E, Okeibunor J, et al. Advocacy for free maternal and child health care in Nigeria--Results and outcomes. Health Policy 2011;99:131-8

9 Yates R. Universal health care and the removal of user fees. The Lancet 2009;373:2078-81.

10 Witter S. Mapping user fees for health care in high-mortality countries-evidence from a recent survey, 2010.

11 Leone T, Cetorelli V, Neal S, et al. Financial accessibility and user fee reforms for maternal healthcare in five sub-Saharan countries: a quasi-experimental analysis. BMJ Open 2016;6:e009692.

12 Dzakpasu S, Soremekun S, Manu A, et al. Impact of free delivery care on health facility delivery and insurance coverage in Ghana's Brong Ahafo region. PLoS One 2012;7:e49430.

13 Ghana Statistical Service GHSG, ICF International. Ghana demographic and health survey 2014, 2015.

14 Mosley WH, Chen LC. An analytical framework for the study of child survival in developing countries. Popul Dev Rev 1984;10:25-45.

15 Fuentes R, Pfütze T, Seck P. Does access to water and sanitation affect child survival? In: A five country analysis: human development report office (HDRO), United nations development programme, 2006.

16 Ridgeway G, Kovalchik SA, Griffin BA, et al. Propensity score analysis with survey weighted data. $J$ Causal Inference 2015;3:237-49.

17 Jann B. Kernel matching with automatic bandwidth selection, 2017.

18 Stuart EA, Green KM. Using full matching to estimate causal effects in nonexperimental studies: examining the relationship between adolescent marijuana use and adult outcomes. Dev Psychol 2008;44:395-406.

19 Stuart EA, Huskamp HA, Duckworth K, et al. Using propensity scores in difference-in-differences models to estimate the effects of a policy change. Health Serv Outcomes Res Methodol 2014;14:166-82.

20 Lunceford JK, Davidian M, MJSim D. Stratification and weighting via the propensity score in estimation of causal treatment effects: a comparative study. Stat Med 2004;23:2937-60.

21 McKinnon B, Harper S, Kaufman JS, et al. Removing user fees for facility-based delivery services: a difference-in-differences evaluation from ten sub-Saharan African countries. Health Policy Plan 2015;30:432-41.

22 Adams AM, Madhavan S, Simon D. Women's social networks and child survival in Mali. Soc Sci Med 2002;54:165-78.

23 Darmstadt GL, Bhutta ZA, Cousens S, et al. Evidence-Based, costeffective interventions: how many newborn babies can we save? Lancet 2005;365:977-88.

24 Browne JL, Kayode GA, Arhinful D, et al. Health insurance determines antenatal, delivery and postnatal care utilisation: evidence from the Ghana demographic and health surveillance data. BMJ Open 2016;6:e008175.

25 Lagarde M, Palmer $\mathrm{N}$. The impact of user fees on access to health services in low-and middle-income countries. Cochrane Database Syst Rev 2011;4.

26 Ansah EK, Narh-Bana S, Asiamah S, et al. Effect of removing direct payment for health care on utilisation and health outcomes in Ghanaian children: a randomised controlled trial. PLoS Med 2009;6:e1000007.

27 Campbell OMR, Graham WJ, LMSSs group. Strategies for reducing maternal mortality: getting on with what works. The Lancet 2006;368:1284-99

28 Corsi DJ, Neuman M, Finlay JE, et al. Demographic and health surveys: a profile. Int J Epidemiol 2012;41:1602-13.

29 Jakubowski A, Stearns SC, Kruk ME, et al. The US president's malaria initiative and under- 5 child mortality in sub-Saharan Africa: a difference-in-differences analysis. PLoS Med 2017;14:e1002319.

30 Munos M, Guiella G, Roberton T, et al. Independent evaluation of the rapid scale-up program to reduce under-five mortality in Burkina Faso. Am J Trop Med Hyg 2016;94:584-95.

31 Zombré D, De Allegri M, Ridde V. Immediate and sustained effects of user fee exemption on healthcare utilization among children under five in Burkina Faso: a controlled interrupted time-series analysis. Soc Sci Med 2017:179:27-35.

32 Sarrassat S, Meda N, Badolo H, et al. Effect of a mass radio campaign on family behaviours and child survival in Burkina Faso: a repeated cross-sectional, cluster-randomised trial. Lancet Glob Health 2018;6:e330-41. 